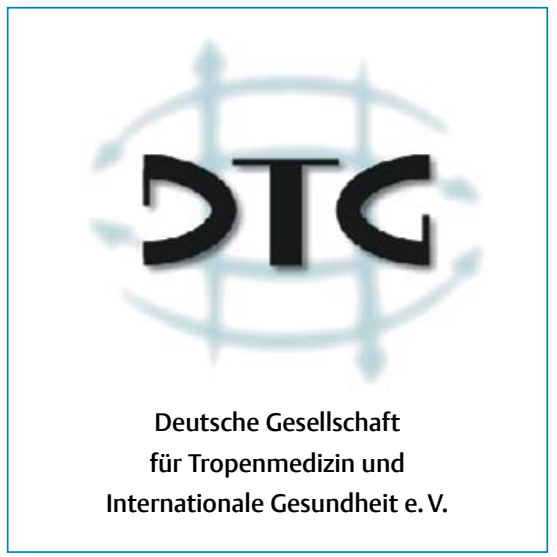

\title{
Liebe Mitglieder und Freunde der DTG,
}

als Tropenmediziner und Internist bin ich in meiner Eigenschaft als Oberfeldarzt des Fachbereichs Tropenmedizin des Bundeswehrkrankenhauses Hamburg Teilnehmer des 1. Kontingents der Bundeswehr im EUTM MALI Einsatz - also der europäischen Trainingsmission für die malische Armee, die so in die Lage versetzt werden soll, den islamistischen Rebellen und Terroristen im Norden des Landes Widerstand zu leisten. Dort, in schwer zugänglichen Regionen, tobt ein von der Presse nicht sehr beachteter, für die französischen Soldaten harter und gefährlicher Krieg unter Wüstenbedingungen; Diarrhö und Mangel an sauberem Wasser und an frischen Lebensmitteln setzen den Soldaten neben dem Gegner schwer zu.

Ich schreibe Ihnen diese Zeilen daher aus dem heißen und staubigen, gespannt auf die Regenzeit wartenden Städtchen Koulikoro. Unser Standort ist eine Offiziersschule der malischen Armee in diesem circa $60 \mathrm{~km}$ entfernt von der Hauptstadt Bamako am Niger liegenden Straßenort mit immerhin 43000 Einwohnern, der laut Wikipedia mit Bous im Saarland verschwistert ist. Die zum Teil leeren Gebäude werden für die Ausbilder und ihre Soldaten hergerichtet, vor wenigen Tagen sind die ersten 600 Rekruten hier angekommen.

Unsere Klinik ist eine kleine, unter sehr beengten räumlichen Bedingungen errichtete Zeltstadt mit den Versorgungsmöglichkeiten einer kleinen, aber gut ausgerüsteten Notfallklinik. Trotz der extremen Hitze und sehr anstrengenden äußeren Bedingungen haben wir keine wesentlichen gesundheitlichen Probleme bisher erlebt; nicht zu vernachlässigen ist aber der Stress durch die Enge, die Einfachheit der Unterbringung und den ständigen Lärm der Generatoren und Klimaanlagen, die ziemlich überfordert sind und mit der schlechten Qualität des einheimischen Diesels zu kämpfen haben. Präventive und reisemedizinische Aspekte sind hier reichlich zu studieren

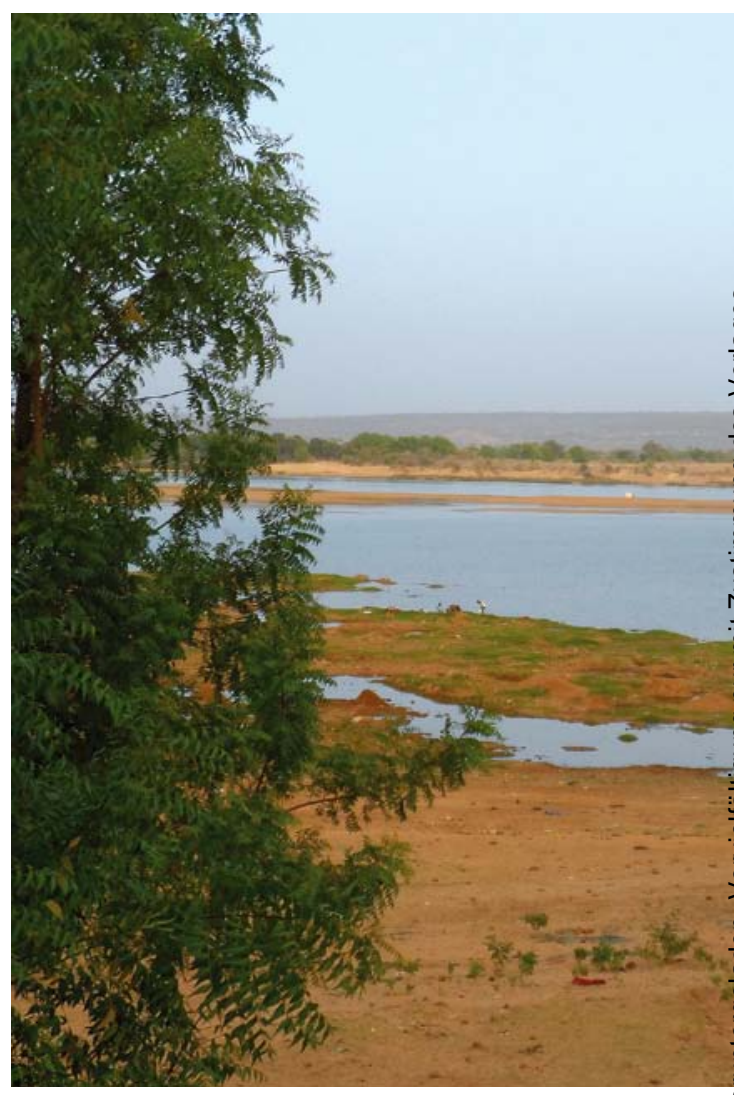

und vor allem auch die Schere zwischen Theorie und Praxis in der Beratung der neu angekommenen Soldaten nicht zu groß werden zu lassen bei den Briefings ist eine ganz interessante Aufgabe. Die ersten Echisvipern-Arten sind erlegt zu uns gebracht worden und nach dem ersten zarten „Mangoregen“ (wie die ersten Schauer überall in Westafrika heißen) nehmen auch die Moskitos ein wenig zu und wir denken eigentlich alle, dass ein Durchfallsausbruch wohl bald kommen wird. In vielen Aspekten fühle ich mich an meine in Westafrika verbrachten Jahre erinnert, wenn auch Leben, Aufgabe und Dresscode sehr viel anders waren... Gleich allerdings die freundliche und gelassene Art der Menschen hier, die überwiegend vom Stamm der Bambara sind, einen offenbar liberalen Islam praktizieren und in der Vergangenheit, wie zum Beispiel 


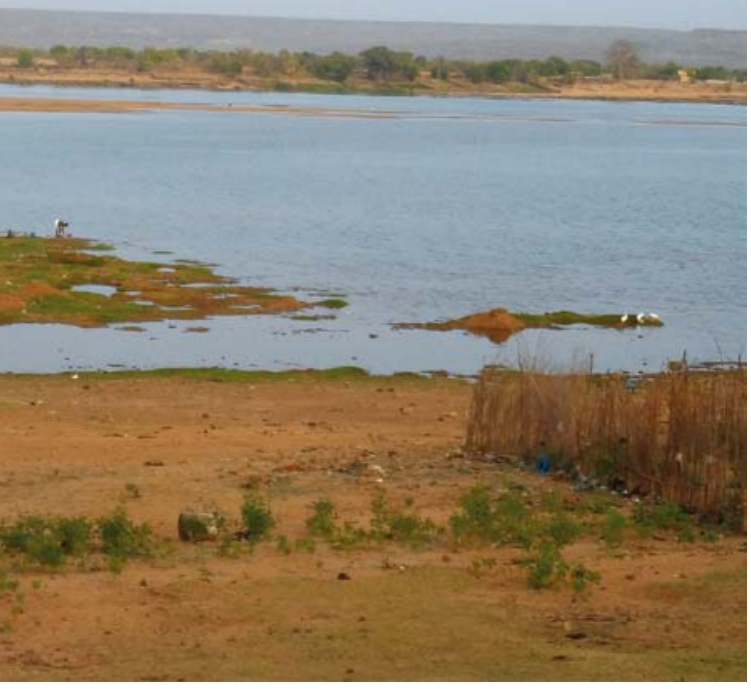

auch die Dogons, einer Hochkultur angehörten, die für ihre Holzschnitzereien berühmt war.

Beitrag und interner Zugang Homepage Kurz vor meiner Ausreise haben Elke Werner und ich noch den folgenden Brief an Sie alle entworfen, der Ende Mai/Anfang Juni an Sie abgeschickt wird:

\section{Sehr geehrte Mitglieder der DTG}

wie bereits auf der letzten Mitgliederversammlung beschlossen und in der FTR mitgeteilt, muss unsere Gesellschaft den Mitgliedsbeitrag moderat erhöhen. Dieses soll vor allem einer verbesserten Serviceleistung und einer Erweiterung des Angebots auf unserer Homepage dienen. Selbstverständlich werden wir auch in Zukunft so sparsam wie möglich mit Ihren Geldern umgehen. Wir hoffen, dass Sie für diese Maßnahme Verständnis haben und wer- den entsprechend zum nächsten Fälligkeitszeitpunkt einen Beitrag von 50 Euro einziehen. Sollten Sie damit nicht einverstanden sein und die Mitgliedschaft aus diesem Grund nicht fortführen wollen (was wir bedauern würden!), bitten wir um baldige Nachricht.

Mit diesem Brief werden wir Ihnen auch Ihre Mitgliedsnummer zusenden, mit der Sie in Zukunft die Seiten der Homepage betreten können, die Mitgliedern vorbehalten sein werden - wie zum Beispiel unser Forum.

Wir hoffen sehr, dass Sie alle uns weiterhin die Treue halten und weiterhin möglichst aktiv am tropenmedizinischen Geschehen teilhaben. Schon bei der Teilnahme an nur einem der von uns mitorganisierten (europäischen) Kongresse sparen Sie als Mitglied der DTG die Beitragserhöhung wieder ein! Der Kongress in Maastricht, www.istm.org, und bald auch der in Kopenhagen, www.ectmih2013.dk, stehen vor der Tür und bieten neben dem interessanten wissenschaftlichen Programm auch viele andere kulturelle, historische und nicht zuletzt kulinarische Höhepunkte - für eine Reservierung im vermeintlich weltbesten Restaurant Noma in Kopenhagen dürfte es allerdings schon zu spät sein... aber im Beluga in Maastricht klappt's vielleicht noch!

Mit herzlichen Grüßen

Thomas Löscher und Hinrich Sudeck

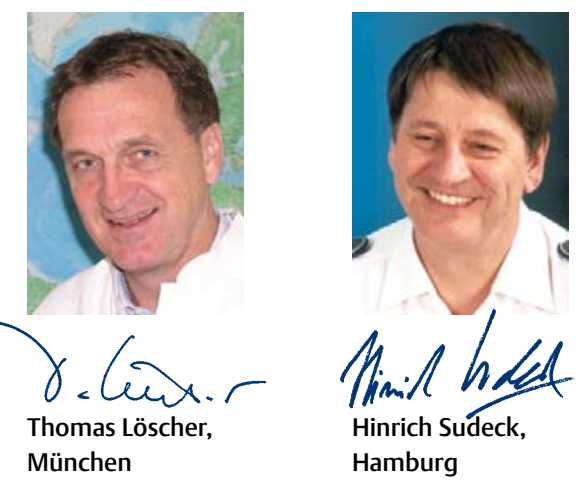

